\title{
Article
}

\section{Nrf2 mediates the resistance of human A549 and HepG2 cancer cells to boningmycin, a new antitumor antibiotic, in vitro through regulation of glutathione levels}

\author{
Hui-xian ZHANG, Yang CHEN, Rong XU, Qi-yang HE* \\ Institute of Medicinal Biotechnology, Chinese Academy of Medical Sciences and Peking Union Medical College, Beijing 100050, China
}

\begin{abstract}
NF-E2-related factor 2 (Nrf2) is a transcription factor and a pivotal factor in the induction of the cell defense system. Recent reports show that Nrf2 plays critical roles in tumor heterogeneity and drug resistance. In the present study we investigated whether and how Nrf2 mediated the resistance of human cancer cells to boningmycin (BON), a new antitumor antibiotic of the bleomycin family. We showed that the expression levels of Nrf2 in human non-small lung cancer A549 cells were much higher than those in human hepatoblastoma HepG2 cells, and their resistance to BON was opposite to Nrf2 expression (the $\mathrm{IC}_{50}$ values of BON in A549 cells and HepG2 cells were 5.97 and $0.61 \mu \mathrm{mol} / \mathrm{L}$, respectively). Similar results were observed with the anticancer agent cisdiamminedichloroplatinum (DDP), which was used as a positive control. In A549 cells, Nrf2 mRNA knockdown significantly increased their susceptibilities to BON and DDP. An enhanced resistance to BON and DDP was observed in HepG2 cells after overexpression of the wild-type Nrf2 protein. Treatment with a specific Nrf2 inhibitor, luteolin, significantly sensitized A549 cells to BON and DDP and increased BON- or DDP-induced apoptosis. The total levels of glutathione (GSH), the final product of the Nrf2 signaling pathway, were much higher in A549 cells than those in HepG2 cells. Supplementation of GSH in HepG2 cells significantly decreased their susceptibility to BON and DDP, wheras depleting GSH with the specific inhibitor L-buthionine sulfoximine in A549 cells significantly increased their susceptibility to BON and DDP. Our results demonstrate that Nrf2 mediates the resistance to BON through regulating glutathione levels in A549 cells and HepG2 cells.
\end{abstract}

Keywords: boningmycin; DDP; Nrf2; drug resistance; glutathione; luteolin; L-buthionine sulfoximine; A549 cells; HepG2 cells

Acta Pharmacologica Sinica (2018) 39: 1661-1669; doi: 10.1038/aps.2018.21; published online 10 May 2018

\section{Introduction}

Boningmycin (BON), a new member of the bleomycin family, was isolated from the fermentation broth of Streptomyces verticillus var. pingyangensis n.sp in China ${ }^{[1]}$. BON has shown higher antitumor activities in vitro and in vivo than bleomycin at the same dose $\mathrm{e}^{[2]}$. To further enhance the translational research of $\mathrm{BON}$, the susceptibility of tumors cells to this drug must be evaluated with biomarkers in the clinic. Investigations using bleomycin have revealed several kinds of cellular proteins that mediate resistance, including bleomycin hydrolase $(\mathrm{BLH})^{[3,4]}$, DNA repair enzymes, antioxidant enzymes, membrane transport proteins and other cellular factors ${ }^{[5-8]}$.

NF-E2-related factor 2 (Nrf2), a transcription factor, is a piv-

\footnotetext{
*To whom correspondence should be addressed. E-mail: qiyang_he@vip.163.com

Received 2017-10-30 Accepted 2018-02-11
}

otal factor in the induction of the cell defense system, primarily including the expression of a myriad of genes involved in oxidant response, phase II detoxification enzymes, ABC transporters and other response pathways ${ }^{[9]}$. Nrf2 protein levels are regulated by several critical regulators, such as the binding partner Keap1 and autophagic pathway proteins p62, p53 and $\mathrm{p} 21^{[10-13]}$. Accumulating evidence has indicated that Nrf2 also serves important roles in tumors. Nrf2 may be a driving force to promote tumorigenesis in several tumor types ${ }^{[14]}$. Constitutive activation of $\mathrm{Nrf} 2$ can result from point mutations in Keap1 in lung carcinoma ${ }^{[15]}$. Nrf2 can also mediate chemoresistance and radioresistance in tumor therapy, and several reports have demonstrated that Nrf2 mediates the resistance to doxorubicin, etoposide and DDP ${ }^{[16-18]}$, suggesting that Nrf2 is a potential drug target for inhibiting tumor growth. Specific compounds, such as luteolin (LUT) and brusatol, reveal effective suppression of tumor cells both in vitro and in vivo ${ }^{[19-21]}$. 
Nrf2 also influences the action and side effects of bleomycin. The degree of pulmonary fibrosis induced by bleomycin was more severe in Nrf2-deficient mice ${ }^{[22]}$. Glutathione (GSH), a downstream product of Nrf2 signaling, markedly reduced bleomycin-induced damage ${ }^{[23,24]}$. Nrf2 mRNA knockdown in A549 cells potentiated bleomycin action ${ }^{[16]}$. These observations may explain why A549 cells exhibited resistance to bleomycin in our previous study ${ }^{[3]}$. We hypothesized that Nrf2 also mediates the resistance to BON. In the present study, we have done several experiments to confirm this hypothesis.

\section{Materials and methods}

Drugs and chemical agents

BON at a purity $>95 \%$ was kindly provided by Prof Ru-xian CHEN from the Institute of Medicinal Biotechnology, Peking Union Medical College and Chinese Academy of Medical Sciences. BON was prepared in a $10 \mathrm{mmol} / \mathrm{L}$ solution with PBS buffer and stored at $-20^{\circ} \mathrm{C}$ before use. Dimethyl sulfoxide (DMSO), 3-(4,5-dimethyl-2-thiazoyl) 2,5-diphenyl-2H-tetrazolium bromide (MTT), L-buthionine-sulfoximine (BSO), glutathione reduced ethyl ester (GSH-M), DDP and LUT were purchased from Sigma-Aldrich Chemical Inc (St Louis, Missouri, USA). BSO was dissolved with PBS buffer in a $150 \mathrm{mmol} / \mathrm{L}$ solution, and DDP was dissolved with DMSO in a $5 \mathrm{mmol} / \mathrm{L}$ solution. LUT was prepared in a $10 \mathrm{mmol} / \mathrm{L}$ solution with DMSO: ethanol $(1: 1, v / v)$ and stored at $-20{ }^{\circ} \mathrm{C}$ before use. The culture media (DMEM and DMEM/F12), monochlorobimane (mBCI), 2,7-dichlorfluorescin diacetate (H2DCFDA), Lipofectamine $^{\mathrm{TM}} 2000$ and fetal bovine serum (FBS) were obtained from Invitrogen (Carlsbad, California, USA).

\section{Cell lines and cell culture}

Human hepatoblastoma HepG2 cells and human non-smallcell lung cancer A549 cells were purchased from Shanghai Cell Bank, Institute of Biochemistry and Cell Biology, Chinese Academy of Sciences. HepG2 cells were cultured in DMEM media, and A549 cells were maintained in DMEM/F12 media. All media were supplemented with $10 \%$ FBS $(v / v)$. All cultures were maintained in an incubator at $37{ }^{\circ} \mathrm{C}$ with $5 \% \mathrm{CO}_{2}$ in a humidified atmosphere.

\section{Cell survival assay}

Cell viability was assessed by MTT assay. The cells were seeded into 96-well plates at a density of 3000 cells per well for $24 \mathrm{~h}$ and then treated with drugs for the indicated times. The MTT assay was performed as previously described ${ }^{[25]}$. The $\mathrm{IC}_{50}$ value was calculated by nonlinear regression analysis.

\section{Western blot analysis}

Western blot analysis was performed as previously described ${ }^{[25]}$. The sources of primary antibodies were as follows. The antibodies against Nrf2 (ab62352) and NQO1 (ab119902) were purchased from Abcam (Abcam, Cambridge, UK). The antibodies against p53 (\#2524S), HO-1 (\#5061S) and $\beta$-actin (\#3700S) were obtained from cell signaling technology
(CST, Danvers, USA). The antibody against BLH (\#MAB6200) was purchased from R\&D Systems (Minnesota, USA). The secondary antibodies, horseradish peroxidase-linked anti-rabbit (\#1705046) and anti-mouse (\#1706516), were obtained from Bio-Rad Ltd (Bio-Rad, California, USA). The levels of $\beta$-actin were used as a loading control.

\section{RNA interference}

Nrf2- and p53-specific siRNAs and scrambled siRNA were purchased from Life technology Inc (Carlsbad, California, USA). Cells were transfected with Lipofectamine 2000 according to the manufacturer's instructions. The final concentration of the siRNAs used for the transfection was 100 pmol. The transfected cells were incubated for $48 \mathrm{~h}$, and the presence of the target protein was confirmed via Western blotting. The Nrf2 siRNA sequence was 5'-GAUGGAAUCUUUGAGGUCAAGUCUA-3'. The p53 siRNA target sequence was 5'-GACTCCAGTGGTAATCTAC-3'.

\section{Plasmid transfection}

Nrf2 gene-carrying plasmids were constructed for this study by our lab. At $70 \%$ to $80 \%$ confluence, HepG2 cells were transfected with the plasmids using Lipofectamine 2000 and then harvested after $48 \mathrm{~h}$ of transfection. The protein levels of Nrf2 were measured by Western blotting.

\section{Total GSH quantification determined by a specific kit}

Cells were sonicated with ultrasonic fragmentation. The volume of the cell lysate was $0.5 \mathrm{~mL}$, and protein concentrations were determined using a Bio-Rad protein assay. The intracellular glutathione concentration was quantified using a Total Glutathione Quantification Kit from Nanjing Jiancheng Bioengineering Institute (Nanjing, China). All procedures were followed according to the manufacturer's instructions. In brief, $50 \mu \mathrm{L}$ of either one of the $50 \mu \mathrm{mol} / \mathrm{L}$ GSH standard solutions or the sample solutions was added to the working buffer. After $30 \mathrm{~s}$, the absorbance was read at $412 \mathrm{~nm}$ (A1) and read once again (A2) after the cuvette was incubated at $25^{\circ} \mathrm{C}$ for 5 $\min$.

Total glutathione $(\mu \mathrm{mol} / \mathrm{L})=(\mathrm{A} 2-\mathrm{A} 1)$ sample $/(\mathrm{A} 2-\mathrm{A} 1)$ standard $50 \mu \mathrm{mol} / \mathrm{L}$

\section{Mean fluorescent intensity of GSH}

The cells were cultured in a $20-\mathrm{mm}$ dish. After drug treatments, the cells were stained with PBS containing $100 \mu \mathrm{mol} / \mathrm{L}$ $\mathrm{mBCI}$ at $37^{\circ} \mathrm{C}$ for $1 \mathrm{~h}$. The fluorescent intensity of individual cells was measured by fluorescence microscopy to detect the intracellular GSH concentration. The mean density from three independent experiments was quantified using Image-Pro Plus software.

\section{Real-time quantitative reverse transcription PCR}

Real-time quantitative reverse transcription-PCR was performed using a SYBR Green PCR kit (Applied Biosystems). Total RNA was extracted using Trizol (Invitrogen), as described by the manufacturer. cDNA was amplified in 28 to 
32 cycles in an ABI 7500 thermal cycler. Amplification of the target genes was normalized using the amplification levels of the endogenous control, GAPDH. The Nrf2 forward primer: 5'-ACACGGTCCACAGCTCATC-3' and reverse primer: 5'-TGTCAATCAAATCCATGTCCTG-3'. NQO1 forward primer: 5'-ATGTATGACAAAGGACCCTTCC-3' and reverse primer: 5'-TCCCTTGCAGAGAGTACATGG-3', HO-1 forward primer: 5'-AACTTTCAGAAGGGCCAGGT-3', and reverse primer: 5'-CTGGGCTCTCCTTGTTGC-3', and GAPDH forward primer: 5'-CTGACTTCAACAGCGACACC-3' and reverse primer: 5'-TGCTGTAGCCAAATTCGTTGT-3', were used for RT-PCR.

Detection of apoptotic cells by Annexin V/propidium iodide staining

The cells were stained with Annexin V and propidium iodide (PI) using an Annexin V-FITC/PI Apoptosis Kit (TransGen, Beijing, China), following the manufacturer's protocol. The fluorescence intensity was measured using a BD FACSCalibur flow cytometer (BD Bioscience, CA, USA).

\section{Detection of reactive oxygen species (ROS)}

The cells were stained with $5 \mu \mathrm{mol} / \mathrm{L} \mathrm{H2DCFDA} \mathrm{(a} \mathrm{specific} \mathrm{dye}$ for ROS) for $30 \mathrm{~min}$ at $37^{\circ} \mathrm{C}$. Cells were washed with PBS three times and immediately analyzed by flow cytometry with excitation and emission settings of $488 \mathrm{~nm}$ and $530 \mathrm{~nm}$, respectively.

\section{Statistical analysis}

Data were expressed as the mean with standard deviations. All experiments were performed three times independently and analyzed by Student's $t$ test. Statistical analyses were performed using SPSS17.0 statistics software with $P<0.05$ as the threshold for statistical significance.

\section{Results}

Resistance to BON in A549 cells with higher Nrf2 protein levels

To explore the relationship between Nrf2 and BON action, we measured the Nrf2 protein levels in A549 and HepG2 cells. Western blot analysis confirmed that Nrf2 was highly expressed in A549 cells (Figure 1A, B), whereas, higher BLH protein levels were detected in HepG2 cells than in A549 cells.

The sensitivities of these cells to BON were determined by MTT assay. The $\mathrm{IC}_{50}$ values of $\mathrm{A} 549$ and HepG2 cells were 5.97 and $0.61 \mu \mathrm{mol} / \mathrm{L}$ (Figure 1C), respectively, revealing BON resistance in A549 cells. Similar results were obtained for the clinical antitumor agent DDP (Figure 1D).

Increased susceptibility to BON by knockdown of endogenous Nrf2 in A549 cells

To further evaluate the role of Nrf2 in BON susceptibility, we knocked down the expression of Nrf2 protein using the RNA interference technique. Western blot analysis revealed that Nrf2 knockdown was maintained after transfection for $72 \mathrm{~h}$.
A

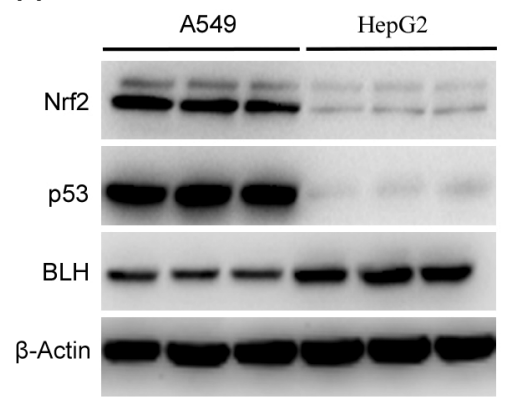

C

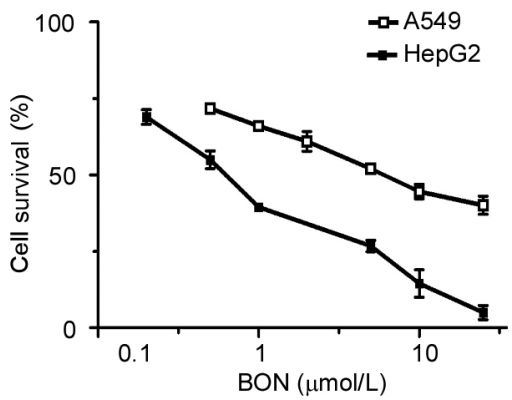

B

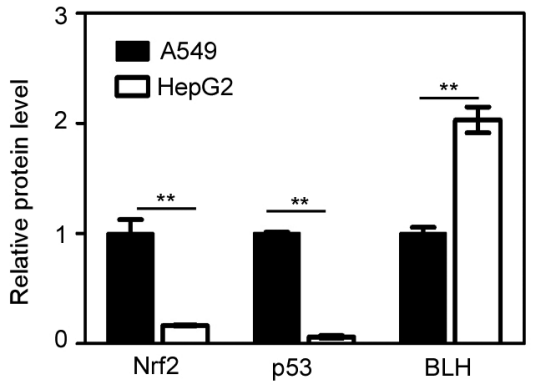

D

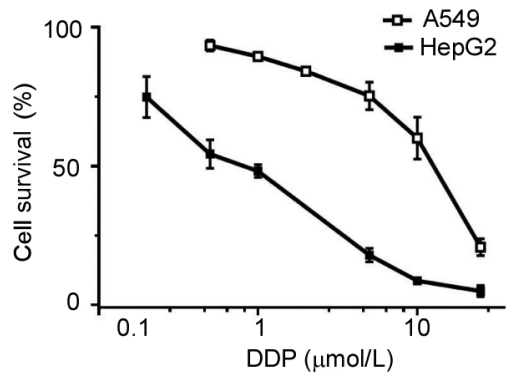

Figure 1. Nrf2 expression and sensitivity to BON and DDP in A549 and HepG2 cells. (A) The protein levels of Nrf2, p53 and BLH were detected by Western blotting. (B) The total protein levels of Nrf2, p53 and BLH were quantified from three independent experiments and represented as the mean \pm SD. ${ }^{* *} P<0.01$ in A549 cells vs HepG2 cells. Cell survival was determined after treatment with BON (C) or DDP (D) for $48 \mathrm{~h}$ and then detected by MTT assay. The results are expressed as the mean \pm SD from three separate experiments. 
NQO1 and HO-1 are downstream enzymes in the Nrf2 signaling pathway, and their expression at both the mRNA and protein level (Figure 2A) was significantly reduced after Nrf2 knockdown, suggesting effective interference of the Nrf2mediated pathway.

Following transfection with Nrf2 siRNA for $24 \mathrm{~h}$, we detected the effect of Nrf2 on the resistance to BON in A549 cells. Cell viability was measured by MTT assay after the cells were treated with the indicated dose of BON or DDP for $48 \mathrm{~h}$ (Figure $2 \mathrm{~B}, \mathrm{C}$ ). The $\mathrm{IC}_{50}$ values of the negative control siRNA (NC-siRNA) and Nrf2 siRNA group were $7.3 \mu \mathrm{mol} / \mathrm{L}$ and $1.9 \mu \mathrm{mol} / \mathrm{L}$, respectively. The sensitivity to $\mathrm{BON}$ in the Nrf2 knockdown group was approximately 4 times greater than that in the NC-siRNA group, indicative of the involvement of Nrf2 in BON susceptibility. A similar phenomenon was observed in the DDP-treated group.

ROS generation caused by BON treatment leads to apopto- sis and cellular senescence in tumor cells ${ }^{[2]}$. Following knockdown of Nrf2 for $24 \mathrm{~h}$, ROS levels were detected to assess the effect of BON after cells were treated with $20 \mu \mathrm{mol} / \mathrm{L} \mathrm{BON}$ for $90 \mathrm{~min}$. The level of ROS in the Nrf2 siRNA group was significantly greater than that in the NC-siRNA group after BON treatment (Figure 2D). These results suggest that reducing Nrf2 protein expression attenuates cellular antioxidant function and enhances the action of BON.

\section{Enhanced resistance to BON by overexpression of Nrf2 in HepG2 cells}

To further confirm the Nrf2-mediated resistance to BON, we constructed a plasmid carrying the wild-type Nrf2 gene. HepG2 cells were transfected with different concentrations of the plasmids, and the overexpression of the Nrf2 protein was measured (Figure 3A). Following transfection with $1 \mu \mathrm{g}$ of the Nrf2 plasmids for $24 \mathrm{~h}$, cell viability to BON or DDP was

A
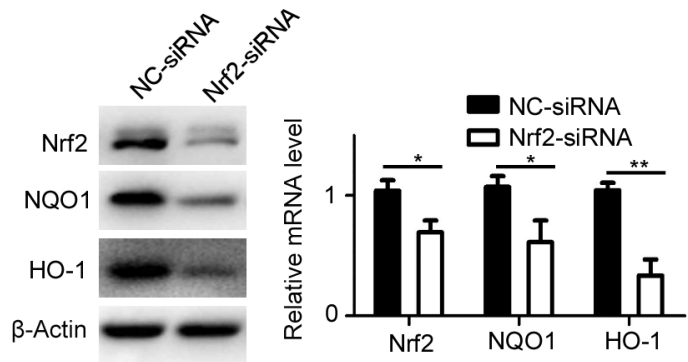

B

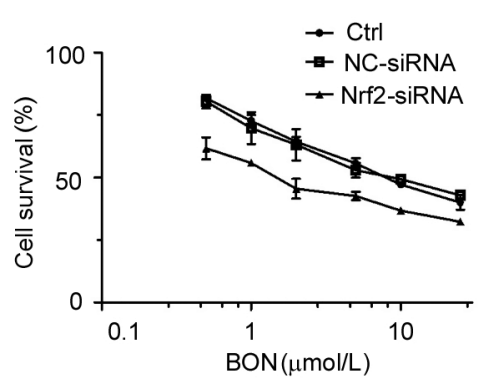

C

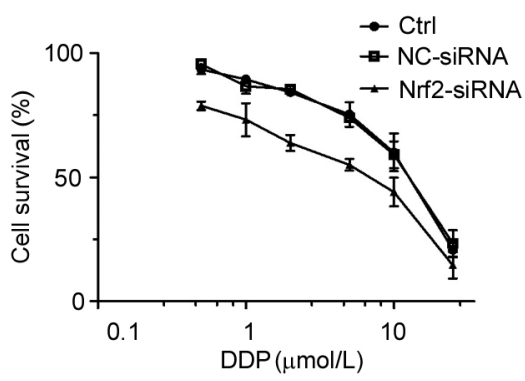

D

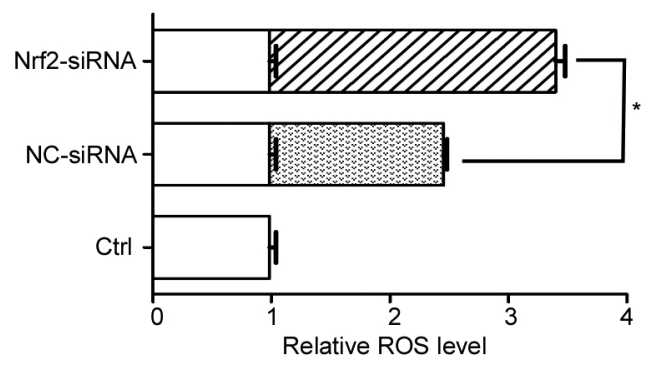

Figure 2. Reduction in Nrf2 expression sensitizes A549 cells to BON and DDP. (A) The protein and mRNA levels of Nrf2, NQO1 and HO-1 were detected after the cells were transfected with Nrf2-siRNA or negative control siRNA for $72 \mathrm{~h}$. The mRNA data were normalized to GAPDH from three independent experiments and represented as the mean \pm SD. Transfected A549 cells had increased susceptibility to BON (B) or DDP (C) after treatment for 48 h. Cell viability was determined by MTT assay. The data are presented as the mean \pm SD. (D) More intracellular ROS was generated in the Nrf2 siRNAtransfected A549 cells after exposure to BON for 90 min than in the BON-treated NC group. 
A
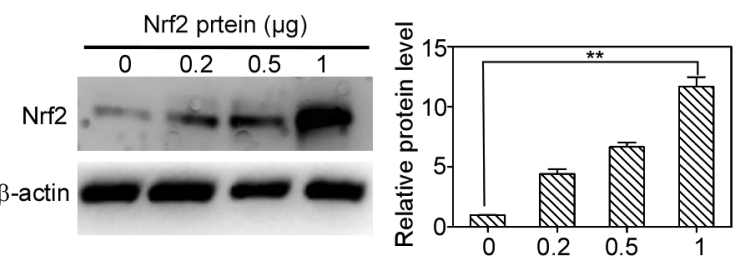

B

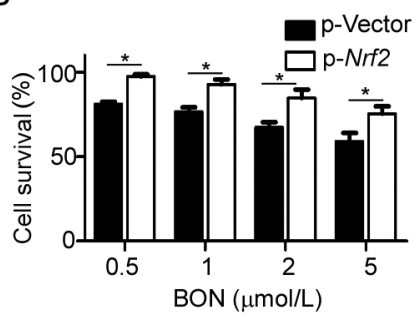

C

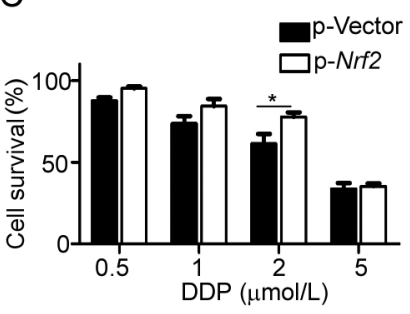

Figure 3. Enhanced resistance to BON in Nrf2-overexpressed HepG2 cells. (A) HepG2 cells were transfected with the Nrf2 gene-carrying plasmid for $48 \mathrm{~h}$ and analyzed by Western blotting. Nrf2 levels were quantified from three independent experiments and represented as the mean \pm SD. Transfected HepG2 cells were treated with BON (B) or DDP (C) for $24 \mathrm{~h}$. Cell viability was determined by MTT assay. Mean \pm SD. ${ }^{*} P<0.05$, ${ }^{* *} P<0.01$.

A

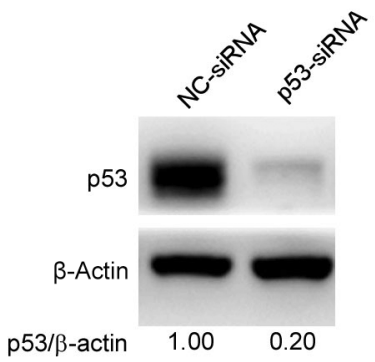

B

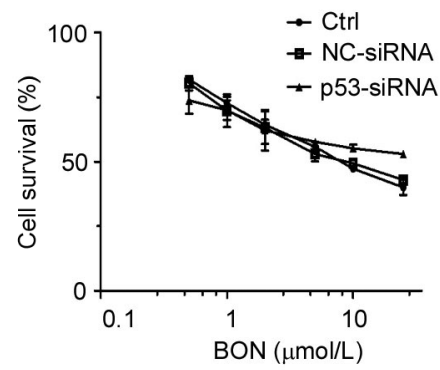

C

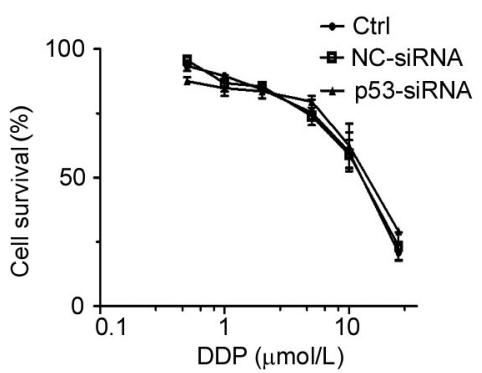

Figure 4. No effect of p53 reduction on sensitivity to BON in A549 cells. (A) The protein expression of p53 in A549 cells was reduced after transfection with p53 siRNA for $72 \mathrm{~h}$. The transfected cells were treated with BON (B) or DDP (C) for $48 \mathrm{~h}$. Cell viability was determined by MTT assay. The data are presented as the mean $\pm \mathrm{SD}$ from two independent experiments.

measured by the MTT assay in HepG2 cells (Figure 3B, C). The resistance to BON sharply increased with Nrf2 protein overexpression, further demonstrating that $\mathrm{Nrf2}$ can mediate $\mathrm{BON}$ resistance.

\section{BON susceptibility was not affected by p53 protein}

Nrf2 expression is regulated by p53 and the direct downstream protein $\mathrm{p} 21^{[11,12]}$. To clarify the effect of $\mathrm{p} 53$ on BON susceptibility in A549 cells, we performed p53 knockdown experiments. The protein levels of p53 were significantly reduced after transfection with p53 siRNA for $72 \mathrm{~h}$ (Figure
4A). However, neither the susceptibility to BON nor DDP was influenced by p53 siRNA (Figure 4B, C), suggesting that p53 does not play a role in regulating the Nrf2-mediated resistance to BON in A549 cells.

\section{Potentiation of BON action by LUT, the Nrf2-specific inhibitor}

Several studies have shown that the flavonoid LUT is a potent Nrf2 inhibitor, as it suppresses Nrf2 activation independently of KEAP1 via a decrease in Nrf2 mRNA expression ${ }^{[20]}$. LUT reduced Nrf2 protein expression and showed almost no suppression of cell proliferation at $10 \mu \mathrm{mol} / \mathrm{L}$ in A549 cells 
(Supplementary Figure S1A, B). The combined treatment of A549 cells with $10 \mu \mathrm{mol} / \mathrm{L}$ LUT and different concentrations of BON or DDP for $48 \mathrm{~h}$ was performed and then analyzed by MTT assay. The results demonstrated that LUT potentiated BON action (Figure 5A), and the synergetic effect of LUT with DDP was greater than that with BON (Figure 5B).

To confirm that the synergy between LUT and BON or DDP was specifically associated with Nrf2, we assayed the cell viability of A549 cells after Nrf2 knockdown. Following transfection with Nrf2 siRNA for $24 \mathrm{~h}$, the cells were treated with LUT alone or in combination with BON for $48 \mathrm{~h}$ (Figure 5C, D). The synergetic action of LUT with BON disappeared, suggesting that the potentiating action of LUT was dependent on Nrf2.

To further identify the potentiating effect of LUT, apoptotic rates were determined after combined treatment with $\mathrm{BON}$ or DDP using Annexin V/PI double staining. In comparison to the proportion of apoptotic cells in those treated with BON or DDP alone, the proportion of apoptotic cells was significantly increased in the combination groups (Figure 5E), indicating that LUT strengthens the drug efficacy by inducing apoptosis in A549 cells.

\section{Regulation of GSH levels by Nrf2 protein levels}

GSH serves an important role in maintaining the redox state, drug detoxification, and antioxidant defense. Nrf2 function is connected with GSH due to the direct induction of $\gamma$-glutamylcysteine ligase expression, the first rate-limiting enzyme in GSH synthesis ${ }^{[26]}$. Quantification of the total GSH levels from the two cell lines revealed that GSH content in A549 cells was four times higher than that in HepG2 cells (Figure 6A). These results were further confirmed by staining with
$\mathrm{mBCl}$, a specific dye for GSH, as the fluorescent intensity was higher in the A549 cells than that in the HepG2 cells (Figure 6B).

Total GSH levels were reduced after the A549 cells were transfected with Nrf2 siRNA for $48 \mathrm{~h}$ (Figure 6C), confirming the association between Nrf2 expression with GSH content. Overexpression of Nrf2 with transfected plasmids elevated GSH levels in the HepG2 cells (Figure 6D). Altogether, these results suggest that GSH levels are regulated by the Nrf2 protein in both cell lines.

\section{BON action affected by GSH}

$\mathrm{BSO}$ is an irreversible inhibitor of $\mathrm{y}$-glutamylcysteine ligase and can deplete cellular GSH levels. A549 cells were treated with different doses of BSO for $24 \mathrm{~h}$, followed by a change in medium and an additional $48 \mathrm{~h}$ of culture. Cell viability was detected by MTT assay. The results showed that $400 \mu \mathrm{mol} / \mathrm{L}$ BSO failed to suppress cell proliferation (Figure 7A) or deplete cellular GSH levels (Figure 7B). A549 cells were then exposed to $400 \mu \mathrm{mol} / \mathrm{L} \mathrm{BSO}$ for $24 \mathrm{~h}$ and then treated with different concentrations of BON or DDP. The cell survival rates decreased from $48.4 \%$ in the group treated with $5 \mu \mathrm{mol} / \mathrm{L}$ $\mathrm{BON}$ to $33.5 \%$ in the group treated with BON plus BSO (Figure $7 \mathrm{C}$ ), and a similar trend was noted in the DDP treatment group (Figure 7D). The results demonstrated that depletion of GSH reduced the resistance of A549 cells to BON.

In contrast, an elevation in GSH levels should increase the resistance to BON. HepG2 cells were pretreated with 4 $\mathrm{mmol} / \mathrm{L}$ GSH-M for $4 \mathrm{~h}$ and then exposed to BON or DDP for $48 \mathrm{~h}$. The sensitivity to BON and DDP decreased after the addition of GSH-M (Figure 8B, C).
A

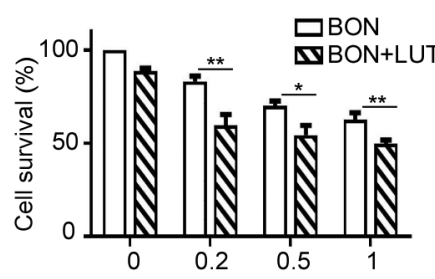

C

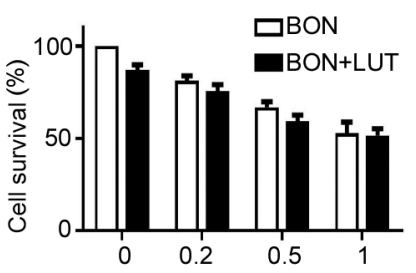

B

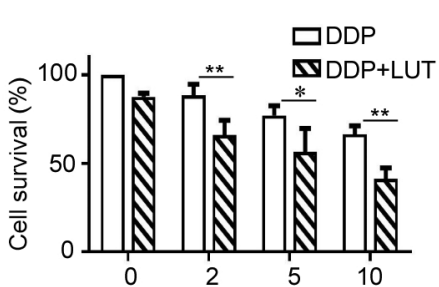

D

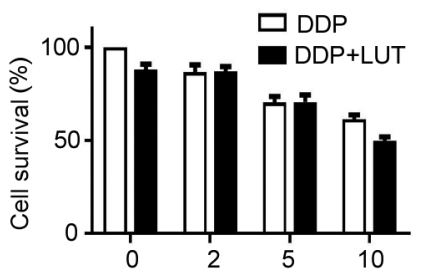

E

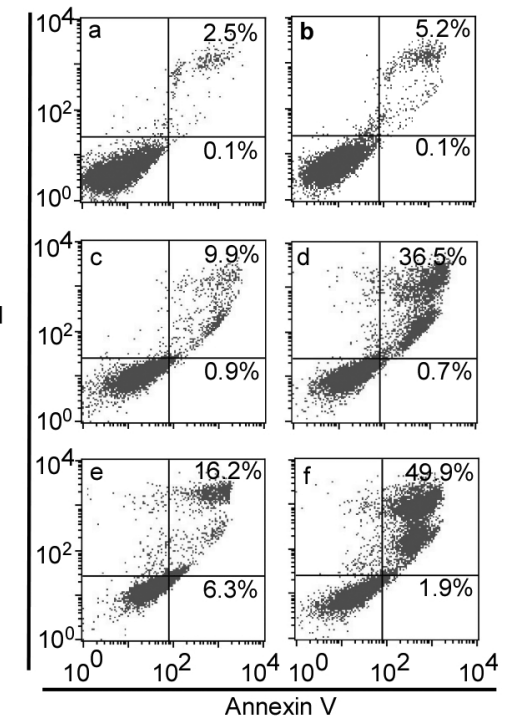

Figure 5. Potentiation of BON action by the Nrf2-specific inhibitor LUT in A549 cells. The A549 cells were treated with BON (A) or DDP (B) in the presence of LUT for $48 \mathrm{~h}$ and analyzed by MTT assay. The Nrf2-reduced A549 cells were treated with BON (C) or DDP (D) in the presence of LUT for $48 \mathrm{~h}$ and analyzed by MTT assay. The results are expressed as the mean \pm SD from three separate experiments. (E) Apoptotic cells were detected by Annexin V/PI staining after treatment with different drugs for 48 h. Control group (a), LUT (b), BON (c), BON+LUT (d), DDP (e), and DDP+LUT (f). 
A

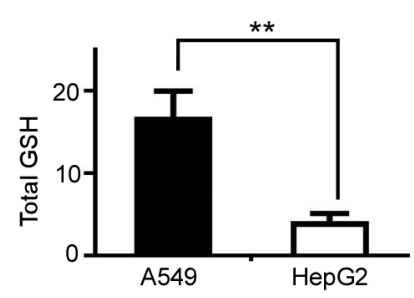

C

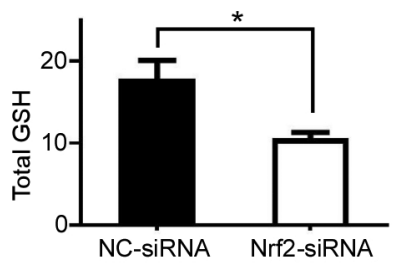

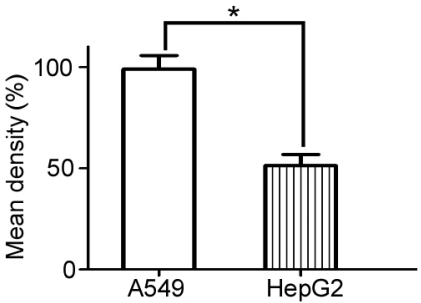

D

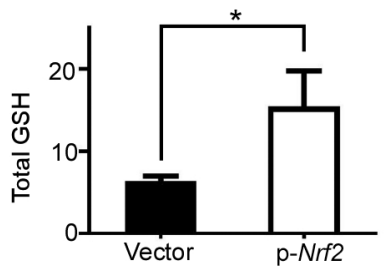

Figure 6. (A) The intracellular GSH levels of A549 cells and HepG2 cells were measured by a GSH assay kit. (B) Cellular GSH levels were determined by the mBCl-stained fluorescent intensity. (C) Reduction in Nrf2 expression decreased intracellular GSH levels. (D) Overexpression of Nrf2 in HepG2 cells increased intracellular GSH levels. ${ }^{*} P<0.05,{ }^{* *} P<0.01$.

A

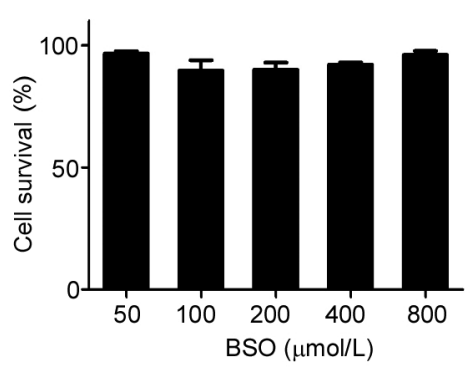

C

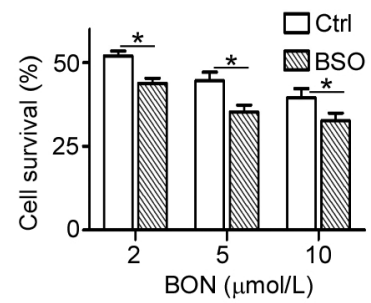

B

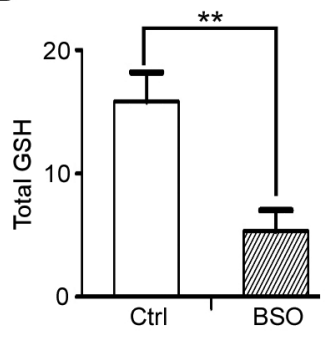

$\mathrm{D}$

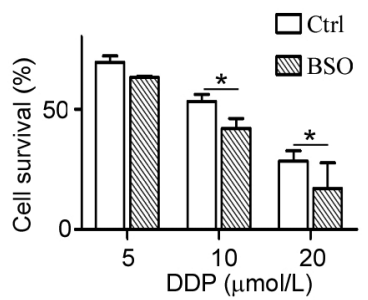

Figure 7. Increased susceptibility to BON by depleting cellular GSH. (A) A549 cells were treated with different concentrations of BSO for $48 \mathrm{~h}$, and cell viability was detected by MTT assay. The data are presented as the mean \pm SD. (B) The intracellular GSH levels in the BSO-treated cells were measured by a GSH assay kit. The A549 cells were pretreated with BSO for $24 \mathrm{~h}$ and then treated with BON (C) or DDP (D) for $48 \mathrm{~h}$ and detected by MTT assay. The results are expressed as the mean \pm SD from three separate experiments. ${ }^{*} P<0.05,{ }^{* *} P<0.01$.

\section{Discussion}

In the present study, we have provided sufficient evidence that Nrf2 mediates resistance to BON, demonstrating that Nrf2 expression can predict BON action in various types of tumors and that BON acts through affecting Nrf2-regulated GSH levels. To the best of our knowledge, this is the first report to demonstrate that the Nrf2 signaling pathway influences the action of BON, a bleomycin family member. Although Nrf2 knockdown via RNA interference was previously shown to increase sensitivity to bleomycin in A549 cells ${ }^{[16]}$, the molecular mechanism connecting Nrf2 protein levels to bleomycin action had not been investigated further. Therefore, the present findings may aid in predicting tumor sensitivity in BON clinical trials, as well as in clinical applications of bleomycin for precise tumor treatments.

Previous studies have indicated that GSH significantly 

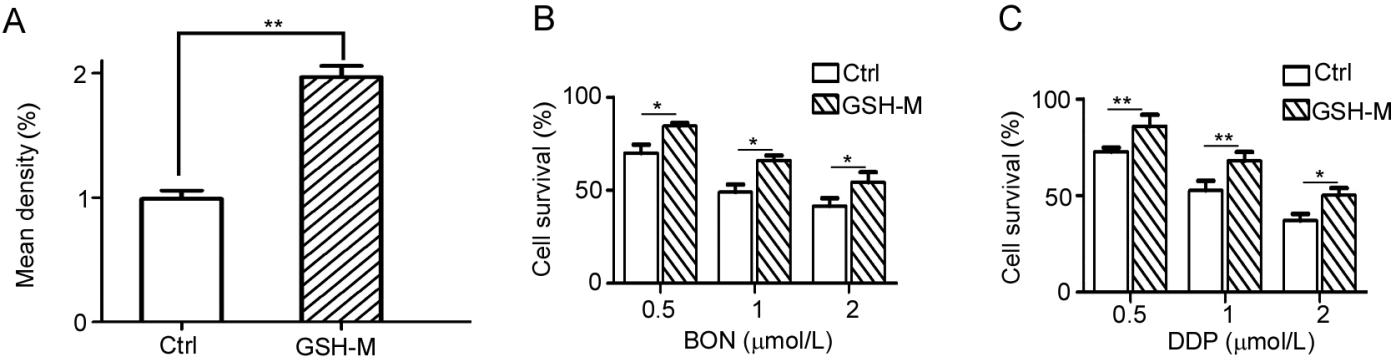

Figure 8. Enhanced resistance to BON by the addition of GSH in HepG2 cells. (A) HepG2 cells were treated with GSH-M for $4 \mathrm{~h}$ and then analyzed by $\mathrm{mBCl}$ staining. The GSH-M-pretreated cells were then treated with BON (B) or DDP (C) for $48 \mathrm{~h}$ and detected by MTT assay. The results are expressed as the mean $\pm \mathrm{SD}$ from three separate experiments.

influences the action of bleomycin. The reduction in GSH induced by BSO treatment has been shown to lead to serious impairments in the DNA repair pathway and an increase in chromosome aberrations in human lymphocytes ${ }^{[23]}$. Although similar in their pattern of DNA damage induced by radiation, the action of BSO differs from that of bleomycin in high linear energy transfer radiation ${ }^{[24]}$. In the present study, the addition of exogenous GSH decreased BON action, suggesting a direct molecular indicator for compounds similar to bleomycin. This information is valuable for investigations of other molecular factors that regulate GSH levels, in addition to Nrf2.

Bleomycin is a first-line antitumor antibiotic that is used in the clinic for several types of malignant tumors, such as malignant lymphoma and testicular cancer ${ }^{[27,28]}$. However, bleomycin induces pulmonary fibrosis, a major side effect that reduces its clinic application ${ }^{[7]}$. Furthermore, commercial bleomycin is a mixture of bleomycin A and bleomycin $B$ that varies based on its production, reducing the efficacy and dose reliability. In contrast, BON has a single chemical structure and a lower induction of pulmonary fibrosis (data not shown), revealing its promise in the treatment of some tumor types in the future. In 2016, a new deglycosylated bleomycin derivative was discovered and was shown to not induce pulmonary fibrosis, while also having potent antitumor effects ${ }^{[2]}$. Therefore, commercial bleomycin will be replaced by new compounds that more precisely treat specific types of cancer with fewer side effects.

To precisely predict the cellular molecules that impact BON action, a systematic investigation of many factors, including Nrf2 and BLH, is required. Although several reports have disputed the influence of BLH on bleomycin action, our previous findings demonstrated that BLH is a bona fide biomarker for determining bleomycin action in some types of cancer ${ }^{[3]}$. BLH protein expression is low in the lungs; however, bleomycin and BON resistance are still found in lung cancer, which may be partly due to high cellular Nrf2 levels. Based on clinical data, approximately $10 \%$ of patients develop pulmonary fibrosis after bleomycin administration ${ }^{[30]}$. The development of pulmonary fibrosis involves Nrf2 function as bleomycin has been shown cause more damage in Nrf2-deficient mice ${ }^{[22]}$. In addition, pulmonary fibrosis is more severe in the homozygous variant G/G of the BLH gene SNP A1450G in testicular gem cell cancer ${ }^{[3]]}$. Investigations into the connection between $\mathrm{BON}$ action and the roles of Nrf2 and BLH are under way in our laboratory.

Recent reports have indicated that Nrf2 plays critical roles in tumor heterogeneity and drug resistance. Nrf2 promotes the survival of cancer stem cells after exposure to $\mathrm{DDP}^{[32]}$. Pancreatic cancers are maintained by Nrf2 through regulating mRNA translation ${ }^{[33]}$. Therefore, Nrf2 is a potential target for the development of antitumor agents. In the present study, the Nrf2-specific inhibitor LUT potentiated the effects of BON and DDP, consistent with previous results ${ }^{[2,34]}$.

In summary, Nrf2 mediates the resistance to BON through regulation of antioxidant GSH levels. Nrf2 may be a valuable target for antitumor action and a biomarker for the precise prediction of drug resistance in some types of tumors.

\section{Acknowledgements}

The work was supported by grants from the National Natural Science Foundation of China (№ 81273553 and 31471150).

\section{Author contribution}

Qi-yang HE designed and guided the study. Hui-xian ZHANG, Yang CHEN, and Rong XU performed the experiments. Hui-xian ZHANG analyzed the data. Qi-yang HE and Hui-xian ZHANG wrote the manuscript. All authors read and approved the final manuscript.

\section{Supplementary information}

Supplementary information is available at the website of Acta Pharmacologica Sinica.

\section{References}

$1 \mathrm{Xu} \mathrm{H}, \mathrm{Yu}$ L, Zhang X, Wang S. Isolation, purification and structure determination of boningmycin (Z-893). J Chin Antibiot 2003; 28: 465-7.

2 Gao N, Shang B, Zhang X, Shen C, Xu R, Xu H, et al. Potent antitumor actions of the new antibiotic boningmycin through induction of apoptosis and cellular senescence. Anticancer Drugs 2011; 22: 16675.

3 Chen J, Chen Y, He Q. Action of bleomycin is affected by bleomycin hydrolase but not by caveolin-1. Int J Oncol 2012; 41: 2245-52.

4 Schwartz DR, Homanics GE, Hoyt DG, Klein E, Abernethy J, Lazo JS. The neutral cysteine protease bleomycin hydrolase is essential for 
epidermal integrity and bleomycin resistance. Proc Natl Acad Sci U S A 1999; 96: 4680-5.

5 Yen HC, Li SH, Majima HJ, Huang YH, Chen CP, Liu CC, et al. Upregulation of antioxidant enzymes and coenzyme $\mathrm{Q}(10)$ in a human oral cancer cell line with acquired bleomycin resistance. Free Radic Res 2011; 45: 707-16.

6 Aouida M, Poulin R, Ramotar D. The human carnitine transporter SLC22A16 mediates high affinity uptake of the anticancer polyamine analogue bleomycin-A5. J Biol Chem 2010; 285: 6275-84.

7 Chen J, Stubbe J. Bleomycins: towards better therapeutics. Nat Rev Cancer 2005; 5: 102-12.

8 Sanz G, Mir L, Jacquemin-Sablon A. Bleomycin resistance in mammalian cells expressing a genetic suppressor element derived from the SRPK1 gene. Cancer Res 2002; 62: 4453-8.

9 Suzuki T, Motohashi H, Yamamoto M. Toward clinical application of the Keap1-Nrf2 pathway. Trends Pharmacol Sci 2013; 34: 340-6.

10 Komatsu M, Kurokawa H, Waguri S, Taguchi K, Kobayashi A, Ichimura $\mathrm{Y}$, et al. The selective autophagy substrate p62 activates the stress responsive transcription factor Nrf2 through inactivation of Keap1. Nat Cell Biol 2010; 12: 213-23.

11 Chen W, Sun Z, Wang XJ, Jiang T, Huang Z, Fang D, et al. Direct interaction between Nrf2 and p21(Cip1/WAF1) upregulates the Nrf2mediated antioxidant response. Mol Cell 2009; 34: 663-73.

12 Faraonio R, Vergara P, Di Marzo D, Pierantoni MG, Napolitano M, Russo T, et al. p53 suppresses the Nrf2-dependent transcription of antioxidant response genes. J Biol Chem 2006; 281: 39776-84.

13 Suzuki T, Yamamoto M. Molecular basis of the Keap1-Nrf2 system. Free Radic Biol Med 2015; 88: 93-100.

14 DeNicola GM, Karreth FA, Humpton TJ, Gopinathan A, Wei C, Frese $\mathrm{K}$, et al. Oncogene-induced Nrf2 transcription promotes ROS detoxification and tumorigenesis. Nature 2011; 475: 106-9.

15 Tao S, Wang S, Moghaddam SJ, Ooi A, Chapman E, Wong PK, et al. Oncogenic KRAS confers chemoresistance by upregulating NRF2. Cancer Res 2014; 74: 7430-41.

16 Homma S, Ishii Y, Morishima Y, Yamadori T, Matsuno Y, Haraguchi N, et al. Nrf2 enhances cell proliferation and resistance to anticancer drugs in human lung cancer. Clin Cancer Res 2009; 15: 3423-32.

17 Singh A, Boldin-Adamsky S, Thimmulappa RK, Rath SK, Ashush H, Coulter J, et al. RNAi-mediated silencing of nuclear factor erythroid-2related factor 2 gene expression in non-small cell lung cancer inhibits tumor growth and increases efficacy of chemotherapy. Cancer Res 2008; 68:7975-84.

18 Singh A, Bodas M, Wakabayashi N, Bunz F, Biswal S. Gain of Nrf2 function in non-small-cell lung cancer cells confers radioresistance. Antioxid Redox Signal 2010; 13: 1627-37.

19 Ren D, Villeneuve NF, Jiang T, Wu T, Lau A, Toppin HA, et al. Brusatol enhances the efficacy of chemotherapy by inhibiting the Nrf2- mediated defense mechanism. Proc Natl Acad Sci U S A 2011; 108: 1433-8.

20 Tang X, Wang H, Fan L, Wu X, Xin A, Ren H, et al. Luteolin inhibits Nrf2 leading to negative regulation of the Nrf2/ARE pathway and sensitization of human lung carcinoma A549 cells to therapeutic drugs. Free Radic Biol Med 2011; 50: 1599-609.

21 Olayanju A, Copple IM, Bryan HK, Edge GT, Sison RL, Wong MW, et al. Brusatol provokes a rapid and transient inhibition of Nrf2 signaling and sensitizes mammalian cells to chemical toxicity-implications for therapeutic targeting of Nrf2. Free Radic Biol Med 2015; 78: 202-12.

22 Kikuchi N, Ishii Y, Morishima Y, Yageta Y, Haraguchi N, Itoh K, et al. Nrf2 protects against pulmonary fibrosis by regulating the lung oxidant level and Th1/Th2 balance. Respir Res 2010; 11: 31.

23 Pujari G, Sarma A, Chatterjee A. The influence of reduced glutathione on chromosome damage induced by X-rays or heavy ion beams of different LETs and on the interaction of DNA lesions induced by radiations and bleomycin. Mutat Res 2010; 696: 154-9.

24 Pujari G, Berni A, Palitti F, Chatterjee A. Influence of glutathione levels on radiation-induced chromosomal DNA damage and repair in human peripheral lymphocytes. Mutat Res 2009; 675: 23-8.

25 Chen Y, Xu R, Chen J, Li X, He Q. Cleavage of bleomycin hydrolase by caspase-3 during apoptosis. Oncol Rep 2013; 30: 939-44.

26 Lu SC. Glutathione synthesis. Biochim Biophys Acta 2013; 1830: 3143-53.

27 Einhorn LH. Curing metastatic testicular cancer. Proc Natl Acad Sci U S A 2002; 99: 4592-5.

28 Hanna NH, Einhorn LH. Testicular cancer-discoveries and updates. N Engl J Med 2014; 371: 2005-16.

29 Burgy O, Wettstein G, Bellaye PS, Decologne N, Racoeur C, Goirand F, et al. Deglycosylated bleomycin has the antitumor activity of bleomycin without pulmonary toxicity. Sci TransI Med 2016; 8: 326ra20.

30 Simpson AB, Paul J, Graham J, Kaye SB. Fatal bleomycin pulmonary toxicity in the west of Scotland 1991-95: a review of patients with germ cell tumours. Br J Cancer 1998; 78: 1061-6.

31 de Haas EC, Zwart N, Meijer C, Nuver J, Boezen HM, Suurmeijer AJ, et al. Variation in bleomycin hydrolase gene is associated with reduced survival after chemotherapy for testicular germ cell cancer. J Clin Oncol 2008; 26: 1817-23.

32 Oshimori N, Oristian D, Fuchs E. TGF- $\beta$ promotes heterogeneity and drug resistance in squamous cell carcinoma. Cell 2015; 160: 96376.

33 Chio II, Jafarnejad SM, Ponz-Sarvise M, Park Y, Rivera K, Palm W, et al. NRF2 promotes tumor maintenance by modulating mRNA translation in pancreatic cancer. Cell 2016; 166: 963-76.

34 Tao S, Wang S, Moghaddam SJ, Ooi A, Chapman E, Wong PK, et al. Oncogenic KRAS confers chemoresistance by upregulating NRF2. Cancer Res 2014; 74: 7430-41. 